Vol. $42(1990) \quad[509-512]$

\title{
LIMITS OF UNBOUNDED SEQUENCES OF CONTINUED FRACTIONS
}

\section{JINGCHENG TONG}

Let $X=\left\{x_{k}\right\}_{k \geqslant 1}$ be a sequence of positive integers. Let $Q_{k}=\left[0 ; x_{k}, x_{k-1}, \ldots, x_{1}\right]$ be the finite continued fraction with partial quotients $x_{i}(1 \leqslant i \leqslant k)$. Denote the set of the limit points of the sequence $\left\{Q_{k}\right\}_{k \geqslant 1}$ by $\Lambda(X)$. In this note a necessary and sufficient condition is given for $\Lambda(X)$ to contain no rational numbers other than zero.

Let $X=\left\{x_{k}\right\}_{k \geqslant 1}$ be a sequence of positive integers. Let $Q_{k}=\left[0 ; x_{k}, x_{k-1}, \ldots, x_{1}\right]$ be the finite continued fraction with partial quotients $x_{i}(1 \leqslant i \leqslant k)$. We denote the set of limit points of the sequence $\left\{Q_{k}\right\}_{k \geqslant 1}$ by $\Lambda(X)$. Recently, Angell [1] proved an interesting result on $\Lambda(X): \Lambda(X)$ contains no rational numbers if the sequence $\left\{x_{k}\right\}_{k \geqslant 1}$ is bounded.

It is easily seen that $0 \in \Lambda(X)$ if and only if $X$ is unbounded. In this note, using the idea in [3], we prove that Angell's result holds for a large family of unbounded sequences if 0 is excluded from $\Lambda(X)$.

We first introduce some new notions.

DEFINITION 1: Let $X=\left\{x_{k}\right\}_{k \geqslant 1}$ be a sequence of positive integers and $N$ be a positive integer. An infinite subsequence $\left\{x_{k_{i}}\right\}_{i \geqslant 1}$ is said to be an $N$-subsequence if $x_{k_{i}}=N$ for all sufficiently large $i$.

Definition 2: Let $X=\left\{x_{k}\right\}_{k \geqslant 1}$ be a sequence of positive integers. Then $X$ is said to be an $\mathcal{N}$-sequence if for each $N$-subsequence $\left\{x_{k_{i}}\right\}_{i \geqslant 1}$, the subsequence $\left\{x_{k_{i}-1}\right\}_{i \geqslant 1}$ is bounded, that is, there is a positive number $I(N)$ such that $x_{k_{i}-1} \leqslant I(N)$ for $i=1,2, \ldots$.

Obviously a bounded sequence is an $\mathcal{N}$-sequence. The converse is not true. The following example is an unbounded $\mathcal{N}$-sequence.

EXAMPLE 1: $X=\left\{x_{k}\right\}_{k \geqslant 1}=\{1,1,2,1,8,4,2,1,512,256,128,64,8,4,2,1, \ldots\}$, where $x_{k}=1$ for $k=2^{n},(n=0,1,2, \ldots)$ and $x_{k}=2^{i}$ for $k=2^{n}-i,(n=2,3, \ldots$ and $\left.1 \leqslant i<2^{n-1}\right)$. Because for each $2^{i}$-subsequence $\left\{x_{k_{i}}\right\}$, the subsequence $\left\{x_{k_{i}-1}\right\}$ is bounded by $I\left(2^{i}\right)=2^{i+1}, X$ is an $\mathcal{N}$-sequence.

Now we give the main result.

Received 4 August, 1989

Copyright Clearance Centre, Inc. Serial-fee code: 0004-9729/90 \$A2.00+0.00. 
THEOREM 1. $\Lambda(X)$ contains no rational numbers other than zero if and only if $X$ is an $\mathcal{N}$-sequence.

Proof: Necessity. Suppose $X$ is not an $\mathcal{N}$-sequence. Then there is an $N$ subsequence $\left\{x_{k_{i}}\right\}_{i \geqslant 1}$ such that $\left\{x_{k_{i}-1}\right\}_{i \geqslant 1}$ is not bounded. Hence there is a subsequence $\left\{x_{k_{i_{m}}-1}\right\}_{m \geqslant 1}$ of $\left\{x_{k_{i}-1}\right\}_{i \geqslant 1}$, satisfying $x_{k_{i_{m}}-1} \rightarrow \infty$ as $m \rightarrow \infty$. Since $\left\{x_{k_{i_{m}}}\right\}_{m \geqslant 1}$ is a subsequence of the $N$-subsequence $\left\{x_{k_{i}}\right\}_{i \geqslant 1}$, we have $x_{k_{i_{m}}}=N$ for all sufficiently large $m$. Hence $Q_{k_{i_{m}}} \rightarrow 1 / N$ as $m \rightarrow \infty$. Thus $\Lambda(X)$ contains a rational number other than zero.

Sufficiency. Let $a$ be an arbitrary rational number other than 0 . Since $0<Q_{k}<1$, without loss of generality, we may assume $0<a \leqslant 1$.

We first prove $1 \notin \Lambda(X)$. Suppose there is a subsequence $Q_{k_{i}} \rightarrow 1$ as $i \rightarrow$ $\infty$. Then since $Q_{k_{i}}=\left[0 ; x_{k_{i}}, \ldots, x_{1}\right]<1 / x_{k_{i}},\left\{x_{k_{i}}\right\}_{i \geqslant 1}$ must be a 1 -subsequence. Hence $\left\{x_{k_{i}-1}\right\}_{i \geqslant 1}$ is bounded by $I(1)$ and $Q_{k_{i}}<[0 ; 1, I(1)]=1 /(1+1 / I(1))<1$, a contradiction to the assumption $Q_{k_{i}} \rightarrow 1$. Therefore $1 \notin \Lambda(X)$.

Suppose there is a rational number $a \neq 0$ and $a \in \Lambda(X)$. Since $0<a<1, a$ can be expanded as a finite continued fraction: $a=\left[0 ; a_{1}, \ldots, a_{r}\right]$. Let $Q_{k_{i}} \rightarrow a$. If $\left\{x_{k_{i}}\right\}_{i \geqslant 1}$ is not an $a_{1}$-subsequence, there are infinitely many $i$ such that $x_{k_{i}} \neq a_{1}$. We discuss the following possible cases.

(1) There are infinitely many $i$ with $x_{k_{i}} \geqslant a_{1}+2$. For these $i$, we have

$$
\begin{aligned}
a-Q_{k_{i}} & >\left[0 ; a_{1}, a_{2}, \ldots, a_{r}\right]-\left[0 ; x_{k_{i}}, x_{k_{i}-1}, \ldots, x_{1}\right] \\
& >\left[0 ; a_{1}, 1\right]-\left[0 ; a_{1}+2\right]>1 /\left(a_{1}+2\right)^{2} .
\end{aligned}
$$

Hence $Q_{k_{i}} \nrightarrow a$.

(2) There are infinitely many $i$ with $x_{k_{i}} \leqslant a_{1}-2$. For these $i$, we have

$$
Q_{k_{i}}-a>\left[0 ; a_{1}-2,1\right]-\left[0 ; a_{1}\right]>1 / a_{1}^{2} \text {. }
$$

Hence $Q_{k_{i}} \nrightarrow a$.

(3) There are infinitely many $i$ with $x_{k_{i}}=a_{1}+1$, that is, there is an $\left(a_{1}+1\right)$ subsequence $\left\{x_{k_{i_{m}}}\right\}_{m \geqslant 1}$. Then $\left\{x_{k_{i_{m}}-1}\right\}_{m \geqslant 1}$ is bounded by $I\left(a_{1}+1\right)$ and

$$
a-Q_{k_{i_{m}}}>\left[0 ; a_{1}, 1\right]-\left[0 ; a_{1}+1, I\left(a_{1}+1\right)\right]>1 /\left(1+I\left(a_{1}+1\right)\right)\left(a_{1}+1\right)^{2} .
$$

Hence $Q_{k_{i}} \not a$.

(4) There are infinitely many $i$ with $x_{k_{i}}=a_{1}-1$, that is, there is an $\left(a_{1}-1\right)$ subsequence $\left\{x_{k_{i_{n}}}\right\}_{n \geqslant 1}$. Then there are two possibilities:

(i) There are infinitely many $n$ with $x_{k_{i_{n}}-1} \geqslant 2$. For these $n$, we have

$$
Q_{k_{i_{n}}}-a>\left[0 ; a_{1}-1,2\right]-\left[0 ; a_{1}\right]>1 / 2 a_{1}^{2} .
$$


Hence $Q_{k_{i}} \nrightarrow a$.

(ii) The subsequence $\left\{x_{k_{i_{n}}}-1\right\}_{n} \geqslant 1$ is a 1 -subsequence. Then $\left\{x_{k_{i_{n}}-2}\right\}_{n} \geqslant 1$ is bounded by $I(1)$ and

$$
Q_{k_{i_{n}}}-a>\left[0 ; a_{1}-1,1, I(1)\right]-\left[0 ; a_{1}\right]>1 / a_{1}^{2}(I(1)+1) .
$$

Hence $Q_{k_{i}} \nrightarrow a$.

From the discussion above, we know that $\left\{x_{k_{i}}\right\}_{i \geqslant 1}$ must be an $a_{1}$-subsequence.

Now we prove $x_{k_{i}-(j-1)}=a_{j} \quad(1 \leqslant j \leqslant r)$ for all sufficiently large $i$.

Suppose $j_{0}$ is the smallest index $j$ such that for each $j$ with $1 \leqslant j \leqslant j_{0}$, $\left\{x_{k_{i}-(j-1)}\right\}_{i \geqslant 1}$ is an $a_{j}$-subsequence, but $\left\{x_{k_{i}-j_{0}}\right\}_{i \geqslant 1}$ is not an $a_{j_{0}+1}$-subsequence. Then for sufficiently large $i$, we have

Let

$$
\begin{gathered}
x_{k_{i}-(j-1)}=a_{j} \quad\left(1 \leqslant j \leqslant j_{0}\right), \\
a=\left[0 ; a_{1}, \ldots, a_{j_{0}}, a_{j_{0}+1}, \ldots, a_{r}\right], \\
Q_{k_{i}}=\left[0 ; a_{1}, \ldots, a_{j_{0}}, x_{k_{i}-j_{0}}, \ldots, x_{1}\right] .
\end{gathered}
$$

$$
\begin{aligned}
\alpha_{j_{0}+1} & =\left[a_{j_{0}+1} ; \ldots a_{r}\right], \\
\beta_{j_{0}+1}(i) & =\left[x_{k_{i}-j_{0}} ; \ldots, x_{1}\right], \\
p_{j_{0}} / q_{j_{0}} & =\left[0 ; a_{1}, \ldots, a_{j_{0}}\right] .
\end{aligned}
$$

By a well known fact ([2, Theorem 7.3] or [3, Lemma 1]), we have

$$
\begin{aligned}
a & =\frac{\alpha_{j_{0}+1} p_{j_{0}}+p_{j_{0}-1}}{\alpha_{j_{0}+1} q_{j_{0}}+q_{j_{0}-1}}, \\
Q_{k_{i}} & =\frac{\beta_{j_{0}+1}(i) p_{j_{0}}+p_{j_{0}-1}}{\beta_{j_{0}+1}(i) q_{j_{0}}+q_{j_{0}-1}}, \\
\left|Q_{k_{i}}-a\right| & =\frac{\left|\alpha_{j_{0}+1}-\beta_{j_{0}+1}(i)\right|}{\left(\alpha_{j_{0}+1} q_{j_{0}}+q_{j_{0}-1}\right)\left(\beta_{j_{0}+1}(i) q_{j_{0}}+q_{j_{0}-1}\right)} \\
& >\left|\alpha_{j_{0}+1}^{-1}-\beta_{j_{0}+1}^{-1}\right| /\left(4 q_{j_{0}}^{2}\right) .
\end{aligned}
$$

Consider $D=\left|\alpha_{j_{0}+1}^{-1}-\beta_{j_{0}+1}^{-1}(i)\right|=\left|\left[0 ; a_{j_{0}+1}, \ldots, a_{r}\right]-\left[0 ; x_{k_{i}-j_{0}}, \ldots, x_{1}\right]\right|$. Since $\left\{x_{k_{i}-j_{0}}\right\}_{i \geqslant 1}$ is not an $a_{j_{0}+1}$-subsequence, there are infinitely many $i$ such that $x_{k_{i}-j_{0}} \neq$ $a_{j_{0}+1}$. Similar to the discussion of $x_{k_{i}}$ being an $a_{1}$-subsequence, we may discuss the four cases for infinitely many $i$ : (1) $x_{k_{i}-j_{0}} \geqslant a_{j_{0}+1}+2$, (2) $x_{k_{i}-j_{0}} \leqslant a_{j_{0}+1}-2$, (3) $x_{k_{i}-j_{0}}=a_{j_{0}+1}+1,(4) x_{k_{i}-j_{0}}=a_{j_{0}+1}-1$, and obtain the conclusion $Q_{k_{i}} \nrightarrow a$. Hence $\left\{x_{k_{i}-j_{0}}\right\}_{i \geqslant 1}$ must be an $a_{j_{0}+1}$-subsequence, a contradiction to the assumption. Therefore $x_{k_{i}-(j-1)}=a_{j} \quad(1 \leqslant j \leqslant r)$ for sufficiently large $i$, and

$$
Q_{k_{i}}=\left[0 ; a_{1}, \ldots, a_{r}, x_{k_{i}-r}, \ldots, x_{1}\right] \text {. }
$$


Again we show that $Q_{k_{i}} \nrightarrow a$. We discuss two cases:

(1) $r$ is odd. Then $Q_{k_{i}}>\left[0 ; a_{1}, \ldots, a_{r}, 1\right]=\left(p_{r}+p_{r-1}\right) /\left(q_{r}+q_{r-1}\right)$, and

$$
\left|Q_{k_{i}}-a\right|>\frac{p_{r}}{q_{r}}-\frac{p_{r}+p_{r-1}}{q_{r}+q_{r-1}}>\frac{1}{2 q_{r}^{2}}
$$

(2) $r$ is even. Then $Q_{k_{i}}<\left[0 ; a_{1}, \ldots, a_{r}, 1\right]$ and

$$
\left|Q_{k_{i}}-a\right|>\frac{p_{r}+q_{r-1}}{p_{r}+q_{r-1}}-\frac{p_{r}}{q_{r}}>\frac{1}{2 q_{r}^{2}} .
$$

In both cases, $Q_{k_{i}} \nrightarrow a$. Therefore $\Lambda(X)$ contains no rational number other than 0 . The proof is completed.

\section{REFERENCES}

[1] D. Angell, 'The limiting behaviour of certain sequences of continued fractions', Bull. Austral. Math. Soc. 38 (1988), 67-76.

[2] I. Niven and H.S. Zuckerman, An Introduction to the Theory of Numbers, (3rd edition) (John Wiley and Sons Inc., 1972).

[3] R.T. Worley, 'Estimating $|\alpha-p / q|$ ', J. Austral. Math. Soc. (Series A) 31 (1981), 202-206.

Department of Mathematics and Statistics

University of North Florida

Jacksonville, FL 32216

United States of America 